\title{
Robust Beamforming for SWIPT Systems with Non-linear Energy Harvesting Model
}

\author{
(Invited Paper)
}

\author{
Elena Boshkovska ${ }^{\ddagger}$, Alexander Koelpin ${ }^{\ddagger}$, Derrick Wing Kwan $\mathrm{Ng}^{*}$, Nikola Zlatanov ${ }^{\dagger}$, and Robert Schober ${ }^{\ddagger}$ \\ Friedrich-Alexander-University Erlangen-Nürnberg (FAU), Germany ${ }^{\ddagger}$ \\ The University of New South Wales, Australia* \\ Monash University, Australia ${ }^{\dagger}$
}

\begin{abstract}
This paper investigates resource allocation for simultaneous wireless information and power transfer (SWIPT) downlink systems based on a non-linear energy harvesting model. The resource allocation algorithm design is formulated as a nonconvex optimization problem for the maximization of the total harvested power. The proposed problem formulation not only takes into account imperfect channel state information (CSI) but also guarantees the quality-of-service $(\mathrm{QoS})$ of information transfer. A novel iterative algorithm is proposed to obtain the globally optimal solution of the considered non-convex optimization problem. In each iteration, a rank-constrained semidefinite program (SDP) is solved optimally by SDP relaxation. Simulation results demonstrate the significant gains in harvested power and the robustness against CSI imperfection for the proposed optimal resource allocation, compared to a baseline scheme designed for perfect CSI and the conventional linear energy harvesting model.
\end{abstract}

\section{INTRODUCTION}

The development of the Internet of Things (IoT) has triggered an exponential growth in the number of wireless communication devices worldwide for applications such as environmental monitoring, energy management, and safety management, etc. [1]. In particular, battery powered wireless sensor modules will be unobtrusively and invisibly integrated into clothing, walls, and vehicles, at locations which are inaccessible for wired recharging. The limited lifetime of wireless nodes creates a bottleneck for communication networks. As a result, wireless powered communication was proposed in the literature [2]-[9]. Specifically, wireless communication devices harvest energy from ambient propagating electromagnetic (EM) waves in radio frequency (RF) for extending their lifetimes and supporting the energy consumption required for future information transmission. Besides, wireless channels are broadcast channels which facilitates the possibility of simultaneous wireless information and power transfer (SWIPT) leading to a new paradigm in wireless communication system design.

Recently, the literature has focused on resource allocation algorithm designs that improve the efficiency of various SWIPT systems [4]-[9]. In [4] and [5], resource allocation algorithms were studied for the maximization of the achievable energy efficiency of single-carrier and multi-carrier SWIPT networks, respectively. In [6], by exploiting the extra degrees of freedom offered by multiple antennas, beamforming was proposed to maximize the total transferred wireless power. However, the results in [4]-[6] were based on the overly optimistic assumption of perfect channel state information (CSI). On the other hand, beamforming designs for secure SWIPT networks with the consideration of imperfect CSI were investigated in [7] and [8]

Robert Schober is also with the University of British Columbia. This work was supported in part by the AvH Professorship Program of the Alexander von Humboldt Foundation. for different system settings. However, in most of the literature [2]-[8], resource allocation algorithms were designed based on the assumption of a linear energy harvesting model which was recently shown to be inaccurate and not capable of capturing the non-linear behaviour of RF energy harvesting circuits [9]. Unfortunately, resource allocation algorithms designed for the over simplified linear energy harvesting model may lead to resource allocation mismatches resulting in severe performance degradation. Motivated by the aforementioned prior works, this paper studies the optimal resource allocation algorithm design for SWIPT systems based on a non-linear energy harvesting model, which provides efficient SWIPT despite the imperfect CSI knowledge.

Notation: In this paper, we adopt the following notations. $\mathbf{A}^{H}, \operatorname{Tr}(\mathbf{A})$, and $\operatorname{Rank}(\mathbf{A})$ represent the Hermitian transpose, trace, and rank of matrix $\mathbf{A} ; \mathbf{A} \succeq \mathbf{0}$ indicates that $\mathbf{A}$ is a positive semidefinite matrix; matrix $\mathbf{I}_{N}$ denotes an $N \times N$ identity matrix. $\operatorname{vec}(\mathbf{A})$ denotes the vectorization of matrix $\mathbf{A} . \mathbf{A} \otimes \mathbf{B}$ denotes the Kronecker product of matrices $\mathbf{A}$ and $\mathbf{B}$. $[\mathbf{B}]_{a: b, c: d}$ returns a submatrix of $\mathbf{B}$ including the $a$-th to the $b$-th rows and the $c$-th to the $d$-th columns of $\mathbf{B}$. $[\mathbf{q}]_{m: n}$ returns a vector with the $m$-th to the $n$-th elements of vector q. A complex Gaussian random vector with mean vector $\boldsymbol{\mu}$ and covariance matrix $\boldsymbol{\Sigma}$ is denoted by $\mathcal{C N}(\boldsymbol{\mu}, \boldsymbol{\Sigma})$, and $\sim$ means "distributed as". $\mathbb{C}^{N \times M}$ denotes the space of all $N \times M$ matrices with complex entries. $\mathbb{H}^{N}$ represents the set of all $N$-by- $N$ complex Hermitian matrices. $\mathcal{E}\{\cdot\}$ denotes statistical expectation. $|\cdot|,\|\cdot\|$, and $\|\cdot\|_{F}$ denote the absolute value of a complex scalar, the Euclidean norm, and the Frobenius norm of a vector/matrix, respectively; $\operatorname{Re}\{\cdot\}$ denotes the real part of an input complex number.

\section{SySTEM MODEL}

In this section, we define the channel and energy harvesting models adopted for resource allocation algorithm design.

\section{A. Channel Model}

We consider a flat fading channel for downlink SWIPT systems. The system consists of a transmitter, an information receiver, and $J$ energy harvesting receivers, cf. Figure 1 . The transmitter is equipped with $N_{\mathrm{T}} \geq 1$ antennas. The information receiver is a single-antenna device and each energy harvesting receiver is equipped with $N_{\mathrm{R}} \geq 1$ receive antennas to facilitate energy harvesting. In each time slot, the received signals at the information receiver and energy harvesting receiver $j \in$ $\{1, \ldots, J\}$ are given by

$$
\begin{aligned}
y & =\mathbf{h}^{H}(\mathbf{w} s+\mathbf{v})+n, \text { and } \\
\mathbf{y}_{\mathrm{ER}_{j}} & =\mathbf{G}_{j}^{H}(\mathbf{w} s+\mathbf{v})+\mathbf{n}_{\mathrm{ER}_{j}}, \forall j \in\{1, \ldots, J\},
\end{aligned}
$$




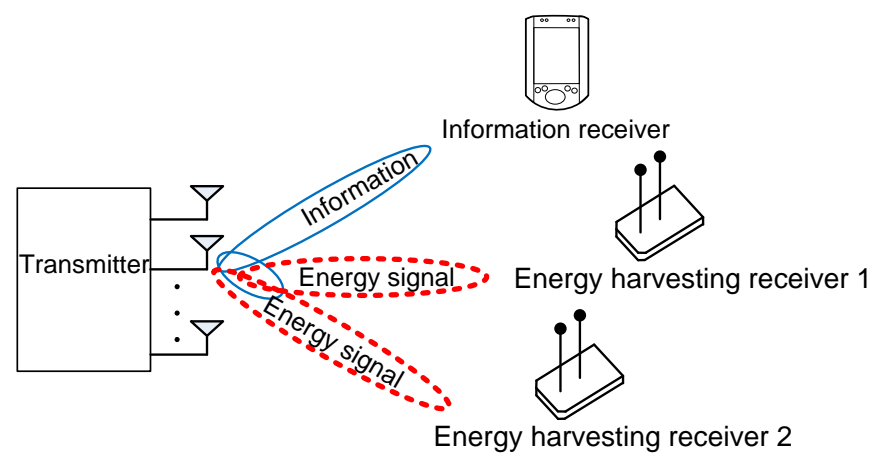

Fig. 1. A downlink SWIPT communication system with an information receiver and $J=2$ energy harvesting receivers (ERs).

respectively, where $s \in \mathbb{C}$ and $\mathbf{w} \in \mathbb{C}^{N_{\mathrm{T}} \times 1}$ are the data symbol and the information beamforming vector, respectively. Without loss of generality, we assume that $\mathcal{E}\left\{|s|^{2}\right\}=1$. $\mathbf{v} \in \mathbb{C}^{N_{\mathrm{T}} \times 1}$ is an energy signal vector generated by the transmitter to facilitate efficient wireless power transfer. $\mathbf{v}$ is modeled as a complex Gaussian random vector with $\mathbf{v} \sim \mathcal{C N}(\mathbf{0}, \mathbf{V})$. The channel vector between the transmitter and the information receiver is denoted by $\mathbf{h} \in \mathbb{C}^{N_{\mathrm{T}} \times 1}$ and the channel matrix between the transmitter and energy harvesting receiver $j$ is denoted by $\mathbf{G}_{j} \in \mathbb{C}^{N_{\mathrm{T}} \times N_{\mathrm{R}}} . n \sim \mathcal{C N}\left(0, \sigma_{\mathrm{s}}^{2}\right)$ and $\mathbf{n}_{\mathrm{ER}} \sim \mathcal{C N}\left(\mathbf{0}, \sigma_{\mathrm{s}}^{2} \mathbf{I}_{N_{\mathrm{R}}}\right)$ are the additive white Gaussian noises (AWGN) at the information receiver and energy harvesting receiver $j$, respectively, where $\sigma_{\mathrm{s}}^{2}$ denotes the noise power at the receiver.

\section{B. Non-linear Energy Harvesting Model}

The total received RF power at energy harvesting receiver $j$ is given by

$$
P_{\mathrm{ER}_{j}}=\operatorname{Tr}\left(\left(\mathbf{w} \mathbf{w}^{H}+\mathbf{V}\right) \mathbf{G}_{j} \mathbf{G}_{j}^{H}\right) .
$$

In practice, an energy harvesting circuit [11]-[13] is equipped at the energy harvesting receiver which is used to convert the received RF power into direct current (DC) power for future use. Yet, practical energy harvesting circuits introduce various nonlinearities into the end-to-end wireless power transfer. In this paper, we adopt a newly proposed non-linear parametric energy harvesting model from [9] for resource allocation algorithm design. In particular, based on experimental results, it has been verified that the parametric non-linear model proposed in [9] is able to accurately capture the dynamics of the RF energy conversion efficiency for different input power levels and the joint effects of the non-linear phenomena caused by hardware imperfections. The total harvested power at energy harvesting receiver $j, \Phi_{\mathrm{ER}_{j}}$, is modelled as:

$$
\begin{aligned}
& \qquad \Phi_{\mathrm{ER}_{j}}=\frac{\left[\Psi_{\mathrm{ER}_{j}}-M_{j} \Omega_{j}\right]}{1-\Omega_{j}}, \Omega_{j}=\frac{1}{1+\exp \left(a_{j} b_{j}\right)}, \\
& \text { where } \Psi_{\mathrm{ER}_{j}}=\frac{M_{j}}{1+\exp \left(-a_{j}\left(P_{\mathrm{ER}_{j}}-b_{j}\right)\right)}
\end{aligned}
$$

is a logistic function which has the received RF power, $P_{\mathrm{ER}_{j}}$, as the input. $M_{j}$ is a constant denoting the maximum harvested power at energy harvesting receiver $j$ when the energy harvesting circuit is saturated because of exceedingly large input power. Parameters $a_{j}$ and $b_{j}$ are constants which capture the joint effects of resistance, capacitance, and circuit sensitivity.
Specifically, $a_{j}$ reflects the non-linear charging rate with respect to the input power and $b_{j}$ is related to the minimum turn-on voltage of an energy harvesting circuit. In practice, parameters $a_{j}, b_{j}$, and $M_{j}$ of the proposed model in (4) can be easily found using a standard curve fitting algorithm for a given energy harvesting hardware circuit.

\section{Channel State Information}

In this paper, we assume that the transmitter has imperfect CSI. To capture the impact of the CSI imperfection on resource allocation design, a deterministic model [14], [15] is adopted. The CSI of the links between the transmitter and the information receiver as well as energy harvesting receiver $j$ can be modelled as:

$$
\begin{aligned}
\mathbf{h} & =\widehat{\mathbf{h}}+\Delta \mathbf{h}, \text { and } \\
\mathbf{\Lambda} & \triangleq\left\{\Delta \mathbf{h} \in \mathbb{C}^{N_{\mathrm{T}} \times 1}:\|\Delta \mathbf{h}\|_{2}^{2} \leq \rho^{2}\right\}, \\
\mathbf{G}_{j} & =\widehat{\mathbf{G}}_{j}+\Delta \mathbf{G}_{j}, \forall j \in\{1, \ldots, J\}, \\
\boldsymbol{\Xi}_{j} & \triangleq\left\{\Delta \mathbf{G}_{j} \in \mathbb{C}^{N_{\mathrm{T}} \times N_{\mathrm{R}}}:\left\|\Delta \mathbf{G}_{j}\right\|_{F}^{2} \leq v_{j}^{2}\right\}, \forall j,
\end{aligned}
$$

respectively, where $\widehat{\mathbf{h}}$ and $\widehat{\mathbf{G}}_{j}$ are the estimates of channel vector $\mathbf{h}$ and matrix $\mathbf{G}_{j}$, respectively. $\Delta \mathbf{h}$ and $\Delta \mathbf{G}_{j}$ represent the channel uncertainty due to channel estimation errors. In (7) and (9), sets $\boldsymbol{\Lambda}$ and $\boldsymbol{\Xi}_{j}$ define continuous spaces spanned by all possible channel uncertainties, respectively. Constants $\rho$ and $v_{j}$ denote the maximum value of the norm of the CSI estimation error vector $\Delta \mathbf{h}$ and matrix $\Delta \mathbf{G}_{j}$, respectively.

\section{Problem Formulation AND SOlution}

The considered system design objective is to maximize the total harvested power while providing QoS for reliable communication with the consideration of imperfect CSI. The resource allocation algorithm design is formulated as the following optimization problem ${ }^{1}$.

$$
\underset{\mathbf{V} \in \mathbb{H}^{N_{\mathrm{T}}}, \mathbf{w}}{\operatorname{maximize}} \quad \sum_{j=1}^{J} \min _{\Delta \mathbf{G}_{j} \in \mathbf{\Xi}_{j}} \Psi_{\mathrm{ER}_{j}}
$$

subject to $\quad \mathrm{C} 1:\|\mathbf{w}\|_{2}^{2}+\operatorname{Tr}(\mathbf{V}) \leq P_{\max }$,

$$
\mathrm{C} 2: \min _{\Delta \mathbf{h} \in \mathbf{\Lambda}} \frac{\mathbf{w}^{H} \mathbf{H w}}{\operatorname{Tr}(\mathbf{V H})+\sigma_{\mathrm{s}}^{2}} \geq \Gamma_{\text {req }}, \quad \mathrm{C} 3: \mathbf{V} \succeq \mathbf{0},
$$

where $\mathbf{H}=\mathbf{h h}^{H}$. Constants $P_{\max }$ and $\Gamma_{\text {req }}$ in constraints $\mathrm{C} 1$ and $\mathrm{C} 2$ are the maximum transmit power and the minimum required signal-to-interference-plus-noise ratio (SINR) at the information receiver, respectively. $\mathrm{C} 3$ and $\mathbf{V} \in \mathbb{H}^{N_{\mathrm{T}}}$ constrain matrix $\mathbf{V}$ to be a positive semidefinite Hermitian matrix. It can be observed that the objective function in (10) is a non-convex function and there are infinitely many inequality constraints in C2. In order to obtain a tractable solution, we first transform the non-convex objective function into an equivalent objective function in subtractive form via the following theorem.

Theorem 1: Suppose $\left\{\mathbf{w}^{*}, \mathbf{V}^{*}\right\}$ is the optimal solution to [10), then there exist two vectors $\boldsymbol{\mu}^{*}=\left[\mu_{1}^{*}, \ldots, \mu_{J}^{*}\right]$ and

\footnotetext{
${ }^{1}$ In the sequel, since $\Omega_{j}$ does not affect the design of the optimal resource allocation policy, with a slight abuse of notation, we will directly use $\Psi_{\mathrm{ER}_{j}}$ to represent the harvested power at ER $j$ for simplicity of presentation.
} 


\section{TABLE I}

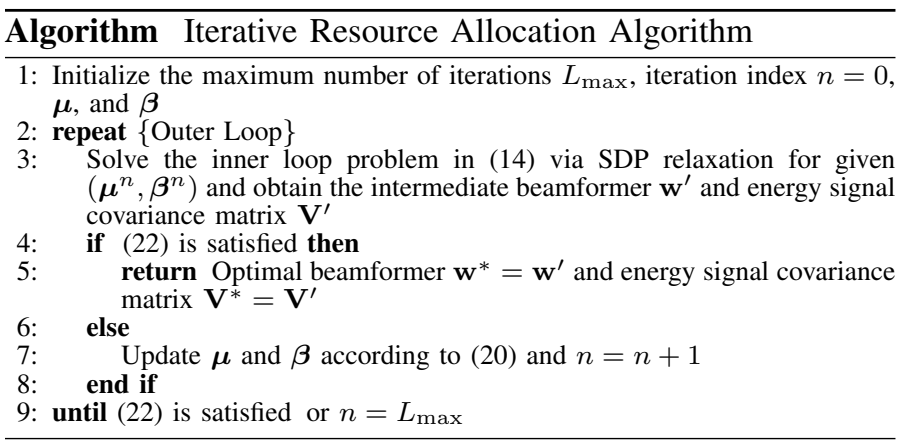

$\boldsymbol{\beta}^{*}=\left[\beta_{1}^{*}, \ldots, \beta_{J}^{*}\right]$ such that $\left\{\mathbf{w}^{*}, \mathbf{V}^{*}\right\}$ is an optimal solution to the following optimization problem

$$
\underset{\mathbf{V}^{*} \in \mathbb{H}^{N_{\mathrm{T}}}, \mathbf{w}^{*} \in \mathcal{F}}{\operatorname{maximize}} \sum_{j=1}^{J} \mu_{j}^{*}\left[M_{j}-\beta_{j}^{*}\left(1+\exp \left(-a_{j}\left(P_{\mathrm{ER}_{j}}-b_{j}\right)\right)\right)\right],
$$

where $\mathcal{F}$ is the feasible solution set of $(10)$. Besides, $\left\{\mathbf{w}^{*}, \mathbf{V}^{*}\right\}$ also satisfies the following system of equations:

$$
\begin{aligned}
\beta_{j}^{*}\left(1+\exp \left(-a_{j}\left(P_{\mathrm{ER}_{j}}^{*}-b_{j}\right)\right)\right)-M_{j} & =0, \\
\mu_{j}^{*}\left(1+\exp \left(-a_{j}\left(P_{\mathrm{ER}_{j}}^{*}-b_{j}\right)\right)\right)-1 & =0,
\end{aligned}
$$

and $P_{\mathrm{ER}_{j}}^{*}=\operatorname{Tr}\left(\left(\mathbf{w}^{*}\left(\mathbf{w}^{*}\right)^{H}+\mathbf{V}^{*}\right) \mathbf{G}_{j} \mathbf{G}_{j}^{H}\right)$.

Proof: Please refer to [16] for a proof of Theorem 1.

As a result, for the maximization problem in [10], there exists an equivalent parametric optimization problem with an objective function in subtractive form and both problems have the same optimal solution $\left\{\mathbf{w}^{*}, \mathbf{V}^{*}\right\}$. More importantly, the optimization problem with an objective function in subtractive form can be solved by an iterative algorithm consisting of two nested loops [16]. In the inner loop, we solve the optimization in (11) for given $(\boldsymbol{\mu}, \boldsymbol{\beta})$. Then, in the outer loop, we find the optimal $\left(\boldsymbol{\mu}^{*}, \boldsymbol{\beta}^{*}\right)$ satisfying the system of equations in (12) and (13), cf. algorithm in Table I]

\section{A. Solution of the Inner Loop Problem}

In each iteration, i.e., line 3 of the algorithm in Table I, we solve the following inner loop non-convex optimization problem:

$$
\begin{array}{ll}
\underset{\mathbf{W}, \mathbf{V} \in \mathbb{H}^{N_{\mathrm{T}}}, \boldsymbol{\tau}}{\operatorname{maximize}} & \sum_{j=1}^{J} \mu_{j}^{*}\left[M_{j}-\beta_{j}^{*}\left(1+\exp \left(-a_{j}\left(\tau_{j}-b_{j}\right)\right)\right)\right] \\
\text { subject to } & \mathrm{C} 1: \operatorname{Tr}(\mathbf{W}+\mathbf{V}) \leq P_{\max }, \\
& \mathrm{C} 2: \min _{\Delta \mathbf{h} \in \mathbf{\Lambda}} \frac{\operatorname{Tr}(\mathbf{W H})}{\operatorname{Tr}(\mathbf{V H})+\sigma_{\mathbf{s}}^{2}} \geq \Gamma_{\text {req }}, \\
& \mathrm{C} 4: \min _{\Delta \mathbf{G}_{j} \in \mathbf{\Xi}_{j}} \operatorname{Tr}\left((\mathbf{W}+\mathbf{V}) \mathbf{G}_{j} \mathbf{G}_{j}^{H}\right) \geq \tau_{j}, \forall j,
\end{array}
$$

C3 : $\mathbf{V} \succeq \mathbf{0}, \mathrm{C} 5: \operatorname{Rank}(\mathbf{W})=1, \mathrm{C} 6: \mathbf{W} \succeq \mathbf{0}$,

where $\mathbf{W}=\mathbf{w} \mathbf{w}^{H}$ is a new optimization variables matrix and $\boldsymbol{\tau}=\left[\tau_{1}, \tau_{2}, \ldots, \tau_{J}\right]$ is a vector of auxiliary optimization variables. To further facilitate the solution, we transform constraints $\mathrm{C} 2$ and $\mathrm{C} 4$ into linear matrix inequalities (LMIs) using the following lemma:

Lemma 1 (S-Procedure [17]): Let a function $f_{m}(\mathbf{x}), m \in$ $\{1,2\}, \mathbf{x} \in \mathbb{C}^{N \times 1}$, be defined as

$$
f_{m}(\mathbf{x})=\mathbf{x}^{H} \mathbf{A}_{m} \mathbf{x}+2 \operatorname{Re}\left\{\mathbf{b}_{m}^{H} \mathbf{x}\right\}+c_{m},
$$

where $\mathbf{A}_{m} \in \mathbb{H}^{N}, \mathbf{b}_{m} \in \mathbb{C}^{N \times 1}$, and $c_{m} \in \mathbb{R}$. Then, the implication $f_{1}(\mathbf{x}) \leq 0 \Rightarrow f_{2}(\mathbf{x}) \leq 0$ holds if and only if there exists a $\delta \geq 0$ such that

$$
\delta\left[\begin{array}{cc}
\mathbf{A}_{1} & \mathbf{b}_{1} \\
\mathbf{b}_{1}^{H} & c_{1}
\end{array}\right]-\left[\begin{array}{cc}
\mathbf{A}_{2} & \mathbf{b}_{2} \\
\mathbf{b}_{2}^{H} & c_{2}
\end{array}\right] \succeq \mathbf{0},
$$

provided that there exists a point $\hat{\mathbf{x}}$ such that $f_{m}(\hat{\mathbf{x}})<0$.

Exploiting Lemma 1, the original constraint $\mathrm{C} 2$ holds if and only if there exists a $\delta \geq 0$, such that the following LMI constraint holds:

$$
\begin{gathered}
\mathrm{C} 2: \mathbf{S}_{\mathrm{C}_{2}}(\mathbf{W}, \mathbf{V}, \delta) \\
=\left[\begin{array}{cc}
\delta \mathbf{I}_{N_{\mathrm{T}}} & \mathbf{0} \\
\mathbf{0} & -\delta \rho^{2}-\Gamma_{\text {req }} \sigma_{\mathrm{s}}^{2}
\end{array}\right]+\mathbf{U}_{\hat{\mathbf{h}}}^{H}\left(\mathbf{W}-\Gamma_{\text {req }} \mathbf{V}\right) \mathbf{U}_{\hat{\mathbf{h}}} \succeq \mathbf{0},
\end{gathered}
$$

where $\mathbf{U}_{\hat{\mathbf{h}}}=\left[\begin{array}{ll}\mathbf{I}_{N_{\mathrm{T}}} & \hat{\mathbf{h}}\end{array}\right]$. Similarly, constraint $\mathrm{C} 4$ can be equivalently written as

$$
\begin{aligned}
& \mathrm{C} 4: \mathbf{S}_{\mathrm{C}_{4_{j}}}(\mathbf{W}, \mathbf{V}, \boldsymbol{\nu}, \boldsymbol{\tau}) \\
= & {\left[\begin{array}{cc}
\nu_{j} \mathbf{I}_{N_{\mathrm{T}} N_{\mathrm{R}}} & \mathbf{0} \\
\mathbf{0} & -\tau_{j}-\nu_{j} v_{j}^{2}
\end{array}\right]+\mathbf{U}_{\widetilde{\mathbf{g}}_{j}}^{H}(\mathcal{W}+\mathcal{V}) \mathbf{U}_{\widetilde{\mathbf{g}}_{j}} \succeq \mathbf{0}, \forall j, }
\end{aligned}
$$

for $\nu_{j} \geq 0, j \in\{1, \ldots, M\}, \mathcal{W}=\mathbf{I}_{N_{\mathrm{R}}} \otimes \mathbf{W}, \mathcal{V}=\mathbf{I}_{N_{\mathrm{R}}} \otimes \mathbf{V}$, $\mathbf{U}_{\widetilde{\mathbf{g}}_{j}}=\left[\begin{array}{ll}\mathbf{I}_{N_{\mathrm{T}} N_{\mathrm{R}}} & \widetilde{\mathbf{g}}_{j}\end{array}\right]$, and $\widetilde{\mathbf{g}}_{j}=\operatorname{vec}\left(\hat{\mathbf{G}}_{j}\right)$. Then, the optimization problem can be equivalently written as

$$
\underset{\mathbf{W}, \mathbf{V} \in \mathbb{H}^{N}, \nu}{\operatorname{maximize}, \delta,} \quad \sum_{j=1}^{J} \mu_{j}^{*}\left[M_{j}-\beta_{j}^{*}\left(1+\exp \left(-a_{j}\left(\tau_{j}-b_{j}\right)\right)\right)\right]
$$

subject to $\mathrm{C} 1, \mathrm{C} 3, \mathrm{C} 6$,

$\mathrm{C} 2: \mathbf{S}_{\mathrm{C}_{2}}(\mathbf{W}, \mathbf{V}, \delta) \succeq \mathbf{0}, \mathrm{C} 4: \mathbf{S}_{\mathrm{C}_{4_{j}}}(\mathbf{W}, \mathbf{V}, \boldsymbol{\nu}, \boldsymbol{\tau}) \succeq \mathbf{0}, \forall j$,

C5 : $\operatorname{Rank}(\mathbf{W})=1, \quad$ C7 $: \nu_{j}, \delta \geq 0$,

where $\delta$ and $\boldsymbol{\nu}=\left\{\nu_{1}, \ldots, \nu_{j}, \ldots, \nu_{J}\right\}$ are the non-negative auxiliary optimization variables introduced by Lemma 1 for handling constraints $\mathrm{C} 2$ and $\mathrm{C} 4$, respectively. We note that constraints $\mathrm{C} 2$ and $\mathrm{C} 4$ involve only a finite number of constraints which facilitates the resource allocation algorithm design. The remaining obstacle in solving the considered optimization problem is the combinatorial rank constraint $\mathrm{C} 5$. We adopt the semidefinite programming (SDP) relaxation by removing constraint C5 from the problem formulation. As a result, the rank constraint relaxed problem becomes a standard convex optimization problem and can be solved efficiently by numerical solvers such as CVX [18]. Yet, the constraint relaxation may not be tight if $\operatorname{Rank}(\mathbf{W})>1$ occurs. Therefore, we reveal the tightness of the adopted SDP relaxation in (10) in the following theorem.

Theorem 2: Assuming the considered problem is feasible for $\Gamma_{\text {req }}>0$, a rank-one solution of (10) can always be constructed.

Proof: Please refer to the Appendix.

In other words, (14) can be solved optimally. Hence, information beamforming is optimal for the maximization of the total harvested power, despite the existence of imperfect CSI.

\section{B. Solution of the Outer Loop Problem}

Now, we present an iterative algorithm to update $(\boldsymbol{\mu}, \boldsymbol{\beta})$ for the outer loop problem via the damped iterative Newton method. For notational simplicity, we define functions $\varphi_{j}\left(\beta_{j}\right)=\beta_{j}\left(1+\exp \left(-a_{j}\left(P_{\mathrm{ER}_{j}}-b_{j}\right)\right)\right)-M_{j}$ and $\varphi_{J+i}\left(\mu_{i}\right)=$ $\mu_{i}\left(1+\exp \left(-a_{i}\left(P_{\mathrm{ER}_{i}}-b_{i}\right)\right)\right)-1, i \in\{1, \ldots, J\}$. It is shown 
TABLE II

SimUlation PARAMETERS

\begin{tabular}{|l|l|}
\hline Carrier center frequency & $915 \mathrm{MHz}$ \\
\hline Bandwidth & $200 \mathrm{kHz}$ \\
\hline Transceiver antenna gain & $12 \mathrm{dBi}$ \\
\hline Noise power & $\sigma^{2}=-95 \mathrm{dBm}$ \\
\hline $\begin{array}{l}\text { Transmitter-to-energy harvesting } \\
\text { receiver fading distribution }\end{array}$ & Rician with Rician factor $3 \mathrm{~dB}$ \\
\hline
\end{tabular}

in [16] that the unique optimal solution $\left(\boldsymbol{\mu}^{*}, \boldsymbol{\beta}^{*}\right)$ is obtained if and only if $\boldsymbol{\varphi}(\boldsymbol{\mu}, \boldsymbol{\beta})=\left[\varphi_{1}, \varphi_{2}, \ldots, \varphi_{2 J}\right]=\boldsymbol{0}$. Therefore, in the $n$-th iteration of the iterative algorithm, $\boldsymbol{\mu}^{n+1}$ and $\boldsymbol{\beta}^{n+1}$ can be updated as, respectively,

$$
\boldsymbol{\mu}^{n+1}=\boldsymbol{\mu}^{n}+\zeta^{n} \mathbf{q}_{J+1: 2 J}^{n} \text { and } \boldsymbol{\beta}^{n+1}=\boldsymbol{\beta}^{n}+\zeta^{n} \mathbf{q}_{1: J}^{n},
$$

where $\mathbf{q}^{n}=\left[\boldsymbol{\varphi}^{\prime}(\boldsymbol{\mu}, \boldsymbol{\beta})\right]^{-1} \boldsymbol{\varphi}(\boldsymbol{\mu}, \boldsymbol{\beta})$

and $\varphi^{\prime}(\boldsymbol{\mu}, \boldsymbol{\beta})$ is the Jacobian matrix of $\boldsymbol{\varphi}(\boldsymbol{\mu}, \boldsymbol{\beta}) . \zeta^{n}$ is the largest $\varepsilon^{l}$ satisfying

$$
\left\|\boldsymbol{\varphi}\left(\boldsymbol{\mu}^{n}+\varepsilon^{l} \mathbf{q}_{J+1: 2 J}^{n}, \boldsymbol{\beta}^{n}+\varepsilon^{l} \mathbf{q}_{1: J}^{n}\right)\right\| \leq\left(1-\eta \varepsilon^{l}\right)\|\boldsymbol{\varphi}(\boldsymbol{\mu}, \boldsymbol{\beta})\|,
$$

where $l \in\{1,2, \ldots\}, \varepsilon^{l} \in(0,1)$, and $\eta \in(0,1)$. The damped Newton method converges to the unique solution $\left(\boldsymbol{\mu}^{*}, \boldsymbol{\beta}^{*}\right)$ satisfying the system of equations (12) and (13), cf. [16].

\section{RESULTS}

In this section, we evaluate the system performance of the proposed optimal resource allocation via simulations. The important simulation parameters are listed in Table II We assume that the information receiver and the $J=10$ energy harvesting receivers are located at 50 meters and 10 meters from the transmitter, respectively. The information receiver requires a minimum SINR of $10 \mathrm{~dB}$. In the sequel, we define the normalized maximum channel estimation errors of energy harvesting receiver $j$ and the information receiver as $\sigma_{\text {est }_{G}}^{2}=\frac{v_{j}^{2}}{\left\|\mathbf{G}_{j}\right\|_{F}^{2}}, \forall j$, and $\sigma_{\text {est }_{h}}^{2}=\frac{\rho^{2}}{\|\mathbf{h}\|_{2}^{2}}=5 \%$. For the non-linear EH circuits, we set $M_{j}=20 \mathrm{~mW}$ which corresponds to the maximum harvested power per energy harvesting receiver. Besides, we adopt $a_{j}=6400$ and $b_{j}=0.003$. We solve the optimization problem in 10 and obtain the average system performance by averaging over different channel realizations.

In Figure 2, we study the average total harvested power versus the maximum channel estimation error $\sigma_{\text {est }_{G}}^{2}$, for different numbers of transmit antennas and resource allocation schemes. The maximum transmit power is $P_{\max }=30 \mathrm{dBm}$ and $N_{\mathrm{R}}=2$. As can be observed, the total harvested power decreases with increasing $\sigma_{\text {est }}^{2}$, since the CSI quality degrades with increasing $\sigma_{\text {est }_{G}}^{2}$. In particular, for a larger value of $\sigma_{\text {est }_{G}}^{2}$, it is more difficult for the transmitter to steer the transmission towards the energy harvesting receivers accurately to improve the efficiency of wireless power transfer. On the other hand, the total harvested power in the system improves with increasing number of transmit antennas. This is because the extra degrees of freedom introduced by additional transmit antennas can be exploited for a more efficient resource allocation. Furthermore, the proposed optimal scheme is able to fulfill the minimum required receive SINR in all considered scenarios, despite the imperfect CSI knowledge.

For comparison, we also show the performance of a baseline scheme. For the baseline scheme, the resource allocation algorithm is designed for the conventional linear energy harvesting model [2]-[8]. Besides, the transmitter of the baseline scheme

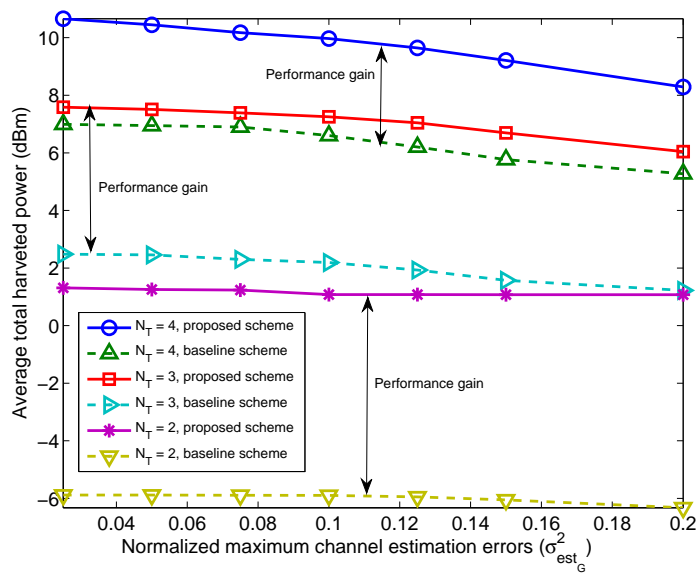

Fig. 2. Average total harvested power $(\mathrm{dBm})$ versus the normalized maximum channel estimation error.

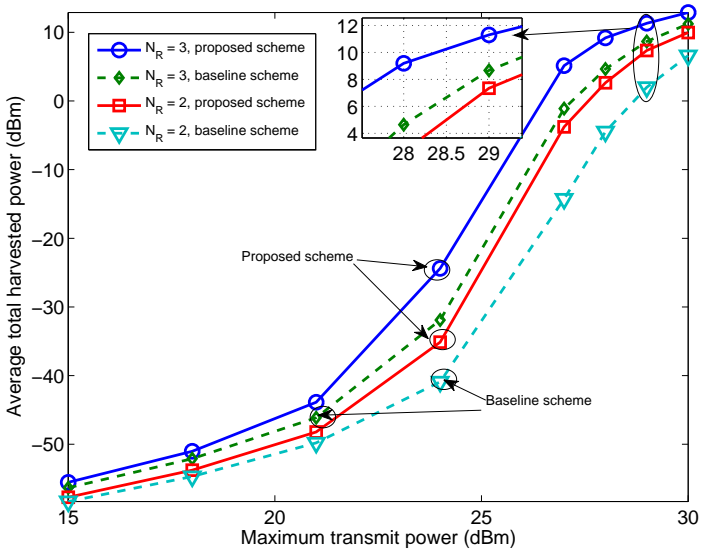

Fig. 3. Average total harvested power $(\mathrm{dBm})$ versus the maximum transmit power $(\mathrm{dBm})$.

treats the estimated channel matrices $\hat{\mathbf{G}}_{j}, \forall j$, as perfect CSI for resource allocation. Then, we optimize the power of $\mathbf{w}, \mathbf{V}$ subject to the constraints in 107 . It can be observed that the proposed optimal algorithm provides a substantial performance gain compared to the baseline scheme, particularly when the estimation errors are comparatively large. In fact, the baseline scheme may cause mismatches in resource allocation since it does not account for the non-linear nature of the energy harvesting circuits.

Figure 3 illustrates the average total harvested power versus the maximum transmit power for different number of receive antennas $N_{\mathrm{R}}$. The normalized maximum channel estimation error is $\sigma_{\text {est }_{G}}^{2}=0.1$ and $N_{\mathrm{T}}=4$. As can be observed, the average total harvested power increases with the maximum transmit power non-linearly. In particular, when the maximum transmit power is small, e.g. $P_{\max } \leq 21 \mathrm{dBm}$, the total harvested power increases slowly with the transmit power. In fact, most of the time, the received power at the energy harvesting receivers is insufficient for switching on the energy harvesting circuits. For a moderate transmit power level, e.g. $21 \leq P_{\max } \leq 27 \mathrm{dBm}$, the total harvested power increases rapidly respect to the transmit power. However, when the transmit power is sufficiently large, e.g. $P_{\max } \geq 27 \mathrm{dBm}$, the total harvested power increases with the maximum transmit power with diminishing return. This is due to the fact that an exceedingly large transmit power causes saturation in some energy harvesting receivers. On the other hand, when the number of antenna equipped at the energy harvesting receivers increases, a significant energy harvesting gain can be achieved by the proposed optimal scheme. In fact, 
the extra receiver antennas act as additional energy collectors which enables a more efficient energy transfer.

\section{Conclusions}

In this paper, we studied the resource allocation algorithm design for SWIPT based on a non-linear energy harvesting model and imperfect CSI. The algorithm design was formulated as a non-convex optimization problem for the maximization of the total power transferred to the energy harvesting receivers. The non-convex optimization problem was solved optimally with an iterative algorithm. Numerical results showed the potential gains in harvested power enabled by the proposed optimization.

\section{APPENDIX-PROOF OF THEOREM 2}

If $\operatorname{Rank}(\mathbf{W})>1$ is obtained from (14), we can construct an optimal rank-one solution as follows. For a given optimal $\boldsymbol{\tau}^{*}$ from the solution of the SDP relaxed version of (14), we solve the following optimization problem:

$$
\begin{array}{cl}
\underset{\mathbf{W}, \mathbf{V} \in \mathbb{H}^{N_{\mathrm{T}}, \delta, \boldsymbol{\nu}}}{\operatorname{minimize}} & \operatorname{Tr}(\mathbf{W}) \\
\text { subject to } & \mathrm{C} 1-\mathrm{C} 3, \mathrm{C} 6, \mathrm{C} 7, \\
& \mathrm{C} 4: \mathbf{S}_{\mathrm{C}_{4_{j}}}\left(\mathbf{W}, \mathbf{V}, \boldsymbol{\nu}, \boldsymbol{\tau}^{*}\right) \succeq \mathbf{0}, \forall j .
\end{array}
$$

We note that the optimal resource allocation policy obtained from (23) is also an optimal resource allocation policy for the SDP relaxed version of (14), since both problems have an identical feasible solution set. Now, we aim to show that 23) admits a rank-one beamforming matrix. To this end, we first need the Lagrangian of problem 23) which is given by:

$$
\begin{aligned}
L & =\operatorname{Tr}(\mathbf{W})+\lambda\left(\operatorname{Tr}(\mathbf{W}+\mathbf{V})-P_{\max }\right)-\operatorname{Tr}(\mathbf{W} \mathbf{Y}) \\
& -\sum_{j=1}^{J} \operatorname{Tr}\left(\mathbf{S}_{\mathrm{C}_{4_{j}}}\left(\mathbf{W}, \mathbf{V}, \boldsymbol{\nu}, \boldsymbol{\tau}^{*}\right) \mathbf{D}_{\mathrm{C}_{4_{j}}}\right) \\
& -\operatorname{Tr}\left(\mathbf{S}_{\mathrm{C}_{2}}(\mathbf{W}, \mathbf{V}, \delta) \mathbf{D}_{\mathrm{C}_{2}}\right)-\operatorname{Tr}(\mathbf{V Z})+\boldsymbol{\Delta},
\end{aligned}
$$

where $\lambda \geq 0, \mathbf{D}_{\mathrm{C}_{2}} \succeq \mathbf{0}, \mathbf{Z} \succeq \mathbf{0}, \mathbf{D}_{\mathrm{C}_{4}} \succeq \mathbf{0}, \forall j \in\{1, \ldots, J\}$, $\mathbf{Y} \succeq \mathbf{0}$, are the dual variables for constraints $\mathrm{C} 1-\mathrm{C} 4$, and $\mathrm{C} 6$, respectively. $\Delta$ is a collection of variables and constants that are not relevant to the proof.

Now, we focus on those Karush-Kuhn-Tucker (KKT) conditions which are needed for the proof:

$$
\begin{aligned}
& \mathbf{Y}^{*}, \mathbf{V}^{*}, \mathbf{D}_{\mathrm{C}_{2}}^{*}, \mathbf{D}_{\mathrm{C}_{4_{j}}}^{*} \succeq \mathbf{0}, \quad \lambda^{*} \geq 0, \\
& \mathbf{Y}^{*} \mathbf{W}^{*}=\mathbf{0}, \quad \mathbf{Q}^{*} \mathbf{V}^{*}=\mathbf{0}, \\
& \mathbf{Y}^{*}=\left(1+\lambda^{*}\right) \mathbf{I}_{N_{\mathrm{T}}}-\mathbf{U}_{\hat{\mathbf{h}}} \mathbf{D}_{\mathrm{C}_{2}} \mathbf{U}_{\hat{\mathbf{h}}}^{H}-\boldsymbol{\Xi} \\
& \mathbf{Q}^{*}=\lambda^{*} \mathbf{I}_{N_{\mathrm{T}}}+\Gamma_{\mathrm{req}} \mathbf{U}_{\hat{\mathbf{h}}} \mathbf{D}_{\mathrm{C}_{2}} \mathbf{U}_{\hat{\mathbf{h}}}^{H}-\boldsymbol{\Xi}, \\
& \mathbf{S}_{\mathrm{C}_{2}}(\mathbf{W}, \mathbf{V}, \delta) \mathbf{D}_{\mathrm{C}_{2}}=\mathbf{0},
\end{aligned}
$$

where $\boldsymbol{\Xi}=\sum_{j=1}^{J} \sum_{l=1}^{N_{\mathrm{R}}}\left[\mathbf{U}_{\widetilde{\mathbf{g}}_{j}} \mathbf{D}_{\mathrm{C}_{4_{j}}} \mathbf{U}_{\widetilde{\mathbf{g}}_{j}}^{H}\right]_{a: b, c: d}, a=(l-1) N_{\mathrm{T}}+$ $1, b=l N_{\mathrm{T}}, c=(l-1) N_{\mathrm{T}}+1$, and $d=l N_{\mathrm{T}}$. The optimal primal and dual variables of the SDP relaxed version are denoted by the corresponding variables with an asterisk superscript.

Then, we follow a similar approach as [7] to show that $\operatorname{Rank}\left(\mathbf{W}^{*}\right)=1$. Subtracting 28 from 27 yields:

$$
\mathbf{Y}^{*}+\left(1+\Gamma_{\text {req }}\right) \mathbf{U}_{\hat{\mathbf{h}}} \mathbf{D}_{\mathrm{C}_{2}} \mathbf{U}_{\hat{\mathbf{h}}}^{H}=\mathbf{Q}^{*}+\mathbf{I}_{N_{\mathrm{T}}} \text {. }
$$

Next, we multiply the both sides of 30 by $\mathbf{W}^{*}$ leading to

$$
\mathbf{W}^{*}\left(1+\Gamma_{\text {req }}\right) \mathbf{U}_{\hat{\mathbf{h}}} \mathbf{D}_{\mathrm{C}_{2}} \mathbf{U}_{\hat{\mathbf{h}}}^{H}=\mathbf{W}^{*}\left(\mathbf{Q}^{*}+\mathbf{I}_{N_{\mathrm{T}}}\right) \text {. }
$$

From (31), we can deduce that

$$
\begin{aligned}
\operatorname{Rank}\left(\mathbf{W}^{*}\right) & =\operatorname{Rank}\left(\mathbf{W}^{*}\left(1+\Gamma_{\operatorname{req}}\right) \mathbf{U}_{\hat{\mathbf{h}}} \mathbf{D}_{\mathrm{C}_{2}} \mathbf{U}_{\hat{\mathbf{h}}}^{H}\right) \\
& \leq \min \left\{\operatorname{Rank}\left(\mathbf{W}^{*}\right), \operatorname{Rank}\left(\left(1+\Gamma_{\operatorname{req}}\right) \mathbf{U}_{\hat{\mathbf{h}}} \mathbf{D}_{\mathrm{C}_{2}} \mathbf{U}_{\hat{\mathbf{h}}}^{H}\right)\right\} .
\end{aligned}
$$

Therefore, if $\operatorname{Rank}\left(\mathbf{U}_{\hat{\mathbf{h}}} \mathbf{D}_{\mathrm{C}_{2}} \mathbf{U}_{\hat{\mathbf{h}}}^{H}\right) \leq 1$, then $\operatorname{Rank}\left(\mathbf{W}^{*}\right) \leq 1$. To show $\operatorname{Rank}\left(\mathbf{U}_{\hat{\mathbf{h}}} \mathbf{D}_{\mathrm{C}_{2}} \mathbf{U}_{\hat{\mathbf{h}}}^{H}\right) \leq 1$, we pre-multiply and postmultiply 29] by $\left[\mathbf{I}_{N_{\mathrm{T}}} \mathbf{0}\right]$ and $\mathbf{U}_{\widetilde{\mathbf{g}}_{j}}^{H}$, respectively. After some mathematical manipulations, we have the following equality:

$$
\begin{aligned}
& \left(\delta \mathbf{I}_{N_{\mathrm{T}}}+\left(\mathbf{W}^{*}-\left(1+\Gamma_{\text {req }}\right) \mathbf{V}^{*}\right)\right) \mathbf{U}_{\hat{\mathbf{h}}} \mathbf{D}_{\mathrm{C}_{2}} \mathbf{U}_{\hat{\mathbf{h}}}^{H} \\
= & \delta[\mathbf{0} \hat{\mathbf{h}}] \mathbf{D}_{\mathrm{C}_{2}} \mathbf{U}_{\hat{\mathbf{h}}}^{H} .
\end{aligned}
$$

Besides, it can be shown that $\left(\delta \mathbf{I}_{N_{\mathrm{T}}}+\left(\mathbf{W}^{*}-\left(1+\Gamma_{\text {req }}\right) \mathbf{V}^{*}\right)\right) \succ \mathbf{0}$ and $\delta>0$ hold at the optimal solution such that the dual optimal solution is bounded from above. Therefore, we have

$$
\begin{aligned}
& \operatorname{Rank}\left(\mathbf{U}_{\hat{\mathbf{h}}} \mathbf{D}_{\mathrm{C}_{2}} \mathbf{U}_{\hat{\mathbf{h}}}^{H}\right) \\
= & \operatorname{Rank}\left(\delta[\mathbf{0} \hat{\mathbf{h}}] \mathbf{D}_{\mathrm{C}_{2}} \mathbf{U}_{\hat{\mathbf{h}}}^{H}\right) \leq \operatorname{Rank}([\mathbf{0} \hat{\mathbf{h}}]) \leq 1 .
\end{aligned}
$$

By combining (32) and (34), we can conclude that $\operatorname{Rank}\left(\mathbf{W}^{*}\right) \leq 1$. On the other hand, since $\Gamma_{\text {req }}>0, \mathbf{W}^{*} \neq \mathbf{0}$ holds and $\operatorname{Rank}\left(\mathbf{W}^{*}\right)=1$.

\section{REFERENCES}

[1] M. Zorzi, A. Gluhak, S. Lange, and A. Bassi, "From Today's INTRAnet of Things to a Future INTERnet of Things: A Wireless- and MobilityRelated View," IEEE Wireless Commun. , vol. 17, pp. 44-51, Dec. 2010.

[2] P. Grover and A. Sahai, "Shannon Meets Tesla: Wireless Information and Power Transfer," in Proc. IEEE Intern. Sympos. on Inf. Theory, Jun. 2010 , pp. $2363-2367$

[3] I. Krikidis, S. Timotheou, S. Nikolaou, G. Zheng, D. W. K. Ng, and R. Schober, "Simultaneous Wireless Information and Power Transfer in Modern Communication Systems," IEEE Commun. Mag., vol. 52, no. 11, pp. 104-110, Nov. 2014.

[4] Q. Wu, M. Tao, D. Ng, W. Chen, and R. Schober, "Energy-Efficient Resource Allocation for Wireless Powered Communication Networks," IEEE Trans. Wireless Commun., vol. PP, no. 99, 2015.

[5] D. W. K. Ng, E. S. Lo, and R. Schober, "Wireless Information and Power Transfer: Energy Efficiency Optimization in OFDMA Systems," IEEE Trans. Wireless Commun., vol. 12, pp. 6352-6370, Dec. 2013.

[6] R. Zhang and C. K. Ho, "MIMO Broadcasting for Simultaneous Wireless Information and Power Transfer," IEEE Trans. Wireless Commun., vol. 12, pp. 1989-2001, May 2013.

[7] M. Khandaker and K.-K. Wong, "Robust Secrecy Beamforming With Energy-Harvesting Eavesdroppers," IEEE Wireless Commun. Lett., vol. 4, pp. 10-13, Feb. 2015.

[8] D. W. K. Ng, E. S. Lo, and R. Schober, "Robust Beamforming for Secure Communication in Systems with Wireless Information and Power Transfer," IEEE Trans. Wireless Commun., vol. 13, pp. 4599-4615, Aug. 2014.

[9] E. Boshkovska, D. Ng, N. Zlatanov, and R. Schober, "Practical Non-Linear Energy Harvesting Model and Resource Allocation for SWIPT Systems," IEEE Commun. Lett., vol. 19, pp. 2082-2085, Dec. 2015.

[10] E. Boshkovska, "Practical Non-Linear Energy Harvesting Model and Resource Allocation in SWIPT Systems," Master's thesis, 2016. [Online]. Available: http://arxiv.org/abs/1602.00833

[11] C. Valenta and G. Durgin, "Harvestıng Wireless Power: Survey of EnergyHarvester Conversion Efficiency in Far-Field, Wireless Power Transfer Systems," IEEE Microw. Mag., vol. 15, pp. 108-120, Jun. 2014.

[12] T. Le, K. Mayaram, and T. Fiez, "Efficient Far-Field Radio Frequency Energy Harvesting for Passively Powered Sensor Networks," IEEE J. Solid-State Circuits, vol. 43, pp. 1287-1302, May 2008.

[13] J. Guo and X. Zhu, "An Improved Analytical Model for RF-DC Conversion Efficiency in Microwave Rectifiers," in IEEE MTT-S Int. Microw. Symp. Dig., June 2012, pp. 1-3.

[14] G. Zheng, K. K. Wong, and T. S. Ng, "Robust Linear MIMO in the Downlink: A Worst-Case Optimization with Ellipsoidal Uncertainty Regions," EURASIP J. Adv. Signal Process., vol. 2008, 2008, Article ID 609028.

[15] D. Ng and R. Schober, "Secure and Green SWIPT in Distributed Antenna Networks With Limited Backhaul Capacity," IEEE Trans. Wireless Commun., vol. 14, pp. 5082-5097, Sep. 2015.

[16] Y. Jong, "An Efficient Global Optimization Algorithm for Nonlinear Sum-of-Ratios Problem," May 2012. [Online]. Available: http://www. optimization-online.org/DBFILE/2012/08/3586.pdf

[17] S. Boyd and L. Vandenberghe, Convex Optimization. Cambridge University Press, 2004

[18] M. Grant and S. Boyd, "CVX: Matlab software for disciplined convex programming, version 2.0 beta," [Online] https://cvxr.com/cvx. Sep. 2012. 